\title{
Multi-Objective Function-Based Node-Disjoint Multipath Routing for Mobile Ad Hoc Networks
}

\author{
Bhanumathi Velusamy ${ }^{1, *(\mathbb{D}, \text { Kalaivanan Karunanithy }}{ }^{2}$, Damien Sauveron ${ }^{3}{ }^{\mathbb{D}}$, Raja Naeem Akram ${ }^{4}$ and \\ Jaehyuk Cho ${ }^{5}$ (1) \\ 1 Department of Electronics and Engineering, Anna University Regional Campus Coimbatore, \\ Coimbatore 641046, Tamilnadu, India \\ 2 School of Electronics Engineering, Vellore Institute of Technology Chennai Campus, \\ Chennai 600127, Tamilnadu, India; kalaivaanankk@yahoo.com \\ 3 MathIS, XLIM, UMR CNRS 7252, Universite de Limoges, 87060 Limoges, France; damien.sauveron@unilim.fr \\ 4 Department of Computer Science, University of Aberdeen, Aberdeen AB24 3FX, UK; raja.akram@abdn.ac.uk \\ 5 School of Electronic Engineering, Soongsil University, Seoul 06978, Korea; chojh@ssu.ac.kr \\ * Correspondence: vbhanu_02@yahoo.com
}

Citation: Velusamy, B.; Karunanithy, K.; Sauveron, D.; Akram, R.N.; Cho, J. Multi-Objective Function-Based Node-Disjoint Multipath Routing for Mobile Ad Hoc Networks. Electronics 2021, 10, 1781. https://doi.org/ 10.3390/electronics10151781

Academic Editors: Paul Mitchell and Martin Reisslein

Received: 29 May 2021

Accepted: 16 July 2021

Published: 25 July 2021

Publisher's Note: MDPI stays neutral with regard to jurisdictional claims in published maps and institutional affiliations.

Copyright: (c) 2021 by the authors. Licensee MDPI, Basel, Switzerland. This article is an open access article distributed under the terms and conditions of the Creative Commons Attribution (CC BY) license (https:/ / creativecommons.org/licenses/by/ $4.0 /)$.

\begin{abstract}
The main goal is to find multiple node-disjoint paths that meet the multi-objective optimization problem in terms of energy consumption minimization and network lifetime improvement. Due to the battery-dependent nodes in mobile ad hoc networks, the performance of the network will degrade. Hence, it is necessary to choose multiple optimal node-disjoint paths between source and destination for data transfer. Additionally, it improves the Quality of Service (QoS) of wireless networks. Multi-objective function is used to select a path such that it gives an optimum result based on the energy consumption, hop, and traffic load. From the simulation results, it is proved that the proposed system is achieving less energy consumption and improved network lifetime compared with existing Dynamic Source Routing (DSR), Hopfield Neural Network-based Disjoint Path set Selection (HNNDPS) and Multipath DSR (MDSR).
\end{abstract}

Keywords: MANET; multipath; neural network; routing; wireless networks

\section{Introduction}

Mobile Ad hoc Network (MANET) is emerging as the most promising application in disaster and emergency communication management. It is very useful where the infrastructure network such as telecom networks fails or there is a network crisis in the case of a disaster such as earthquake, flooding, cyclone, landslide and war etc. MANET provides a solution to the problem and establishes the networks to collect the data without any interruption. Therefore, it is necessary to ensure the reliability of data collection in a rescue operation. MANET is a set of mobile nodes that can dynamically form a network without using any existing centralized administration [1]. It is a communication network in which all nodes are mobile and communicate with each other via wireless connections and can therefore be used as a practical solution for a catastrophe situation [2]. Nodes can join or leave the network at any time, and they communicate with each other within their radio range and communication beyond this range is established by employing intermediate nodes to set up a path in a hop-by-hop manner.

The most difficult aspect of building MANET routing protocols is the energy resource limitation imposed in mobile nodes. Energy-efficient MANET routing algorithms decide how MANET technology can be used for real-time MANET. The mobile nodes consume more power and drain the battery power more quickly due to flaws in central coordination and the dynamic network environment [3]. It is vital to provide energy-efficient routing in MANET, even with frequent changes caused by node mobility, interference, concealed terminal problems, and dead nodes. Each mobile node communicates with the others 
using a multi-hop wireless connection. Every node in a MANET serves as a relay node for receiving and transmitting data from one node to the next. As a result, the intermediate will experience network overload and congestion when the intermediate node is unable to handle network traffic that exceeds its capacity [4,5]. In addition, each transmission in a MANET generates interference, contention, and collisions in its immediate vicinity. The lifetime of the link in MANET is unpredictable because a node's links can be established or disconnected at any time. MANET protocols have been proposed for achieving energy efficiency by load balancing and routing the network traffic [6-10].

Multipath routing protocols have multiple routes, and this can reduce data transmission delays caused by link failure and distributes high traffic load into multiple paths. Node-disjoint and link-disjoint path sets are found with route discovery. Link-disjoint is one if there is no overlapping or common links in the path between a given pair of source and destination nodes in a network, whereas node-disjoint is one in which it does not have common nodes between the source and destination nodes. Many of the existing works in this area focus on how to set up multiple best paths which are node-disjoint [11-13], how to distribute traffic into multiple paths [14], and how to select a path [15]. Power and load-aware routing scheme is based on Dynamic Source Routing (DSR) protocol [12]. Here, sources were allowed to find a multiple node-disjoint paths to destination for maximizing the lifetime of the nodes. An improved performance of the AODV proposed in [16], in which the link expiration time is calculated based on the speed and moving direction of the nodes. Multipath may have common links or nodes. To minimize the node failure in multipath, the node sharing which leads to network partitioning should be avoided. Therefore, it is necessary to choose multiple paths as node-disjoint for our proposed work. Disjoint Path set Selection Protocol (DPSP) given in [17] selects a set of highly reliable paths based on a heuristic. They found link-disjoint paths and reported that almost all the possible paths have been found out by DPSP. Low routing overhead and transmission delays are the advantages of DPSP. The disadvantage is that it can find only link-disjoint paths.

Hopfield Neural Network-based highly reliable Disjoint Path set Selection for mobile ad hoc networks (HNNDPS) is discussed in [18]. Link expiration time is used for finding the link reliability between two nodes. The authors proved that the link-disjoint algorithm is highly reliable compared to node-disjoint. The lifetime is better in node-disjoint than in link-disjoint. The destination node after collecting all the RREQs applies neural network algorithm to the link and node-disjoint path set to select reliable path set. The major disadvantage of this routing is the number of iterations which results in delay in finding multiple paths. In addition, also the reliable path set selection is based on the energy function alone. It does not concentrate on the hop, and traffic load. The nodes in the path set may soon drain out and become unusable.

Multipath DSR (MDSR) given in [19] is to minimize flooding of RREQ and to maintain a backup path to cope with path breakages. Destination gives Route Reply (RREP) only to a selected set of Route Request (RREQ) messages after receiving all route requests. The paths are chosen as link-disjoint. If the selected route is broken, the source node uses an alternative route, which is the shortest among the remaining routes in the cache. This will continue till the route cache is empty or all are broken. Although this method eliminates frequent route discoveries, the alternate paths may be longer and hence the delay per packet increases. In addition to delay, frequent route discoveries may be initiated because only a few intermediate nodes may have alternative paths to a destination. Disjoint MultiPath Source Routing (DMPSR) [20] is designed for achieving minimum overhead using gossipbased route discovery. It makes use of multiple disjoint paths to transmit packets. In gossiping mechanism, a source node initiates by broadcasting the RREQ packet in the network with a probability of $P$ equal to 1 . The rebroadcast is done by other nodes with a probability of $P$ less than or equal to one. Percolation theory is used for determining the probability value. The probability $P$ is chosen such that all nodes can hear a RREQ message in the network. Source collects all RREP messages for finding as many disjoint paths as possible. When the primary route fails, source resumes sending packets using alternative 
paths. Once the communication session is over, destination and other relay nodes are notified about this by the source node and the node can delete the route information from the route cache. Due to multi-hop nature, node's residual energy must be used carefully to avoid route or link failure due to energy drain. Energy consumption should be well managed and minimized to maximize the network lifetime [21].

Many energy-aware routing schemes have been employed to prolong the lifetime of energy constrained mobile nodes in ad hoc networks [14,22-25]. Fuzzy logic-based link stability is determined in [26], in which the best routing path is selected among multiple paths based on the path with highest link stability. Energy reduction in multipath routing in MANET using recoiled node technique is introduced in [27], in which particular nodes only participate in routing the data between the source and destination based on the geographical location and residual energy. Path Discovery and Selection for EnergyEfficient Routing (PDSEER) is designed in [25] which uses Received Signal Strength (RSS) and residual energy to discover the routes. PDSEER ensured that the selected path had high link stability and low energy consumption. Energy-Efficient Bi-objective Path Selection (EE-BPS) is proposed in [28], in which the RSS is used to find the possible paths between the source and destination. Then, it considered the residual energy and hop count to select the best path. Optimal Path Selection Model (OPSM) is proposed in [29], in which the routes are identified with the links with high power ratio and link duration. After that, the source node finds a node-disjoint path depending on the residual energy and hop count. OPSM is aimed to conserve the battery energy by reducing the node failures in the routes. It is stated in [30], the throughput declines as packets traverse a long path and the end-to-end delay increases. Increased hop count leads to increase in the packet loss rate.

The traffic load of a node is defined as the pending amount of traffic in a node's queue. The high traffic load causes a data queue overflow in the nodes, resulting in packet loss. In addition, sensor and ad hoc nodes are battery-dependent and they are quickly exhausted, resulting in the decreased lifetime [31]. Hence, it can be said that the traffic load on the nodes is related to the lifetime of the networks. Therefore, it is necessary to optimize the discovered node-disjoint paths based on the multi-objective optimization criteria for providing energy-efficient communication resulting in the network lifetime enhancement. The multi-objective optimization approach has been applied earlier in all fields such as wireless multicarrier transmission on cognitive radio networks [32] and for secured routing in the ad hoc networks [33]. Optimization is modeled based on the weighted sum approach given in [34] by considering three functions such as energy consumption, hop counts, and traffic load. Therefore, it can be said that energy-efficient path selection can be set up by balancing energy consumption, hop counts, and traffic load. Thus, it can be interpreted that the network partitions occur rarely, and the system can reliably transfer packet through the path.

\subsection{Contribution}

The main contribution in this paper is the proposal of MFNMR, a Multi-objective Function-based Node-disjoint Multipath Routing protocol based on DSR, which aims to find multiple node-disjoint paths that meet the multi-objective optimization problem in terms of energy consumption minimization and network lifetime improvement.

The additional contributions of this paper are:

- Proposing a new energy consumption model to extend the network lifetime.

- Introducing node-disjoint path selection to reduce the interference and enhance the efficiency in terms of energy consumption and Packet Delivery Ratio (PDR).

- Creating multiple node-disjoint paths between source and destination to select the best path.

- Selecting a single optimal path based on the energy consumption, traffic load, and hop count.

- Conducting a comparative evaluation of simulation results of MFNMR against existing Dynamic Source Routing (DSR), Hopfield Neural Network-based Disjoint Path set 
Selection (HNNDPS) and Multipath DSR (MDSR) to show that the proposed system is achieving less energy consumption and improves network lifetime.

- Considering a single path and multipath data transmission to evaluate the performance of the proposed protocol as given in Tables 3 and 4 .

\subsection{Structure of the Paper}

Section 2 describes the problem. Section 3 details the proposed protocol and the energy-efficient node-disjoint source route selection algorithm. Section 4 analyzes the results and provides a discussion. Section 5 concludes this work.

\section{Problem Description}

Previous analysis disclosed that the node-disjoint multipath routing may not be energy-efficient, as there may be a greater number of hops and energy consumption. It is also observed from [19] that the longer alternating paths are less advantageous, because they tend to break too soon. Multi-node joint path often disturbed due to loop formation in the data packets and lead to the link failure. To overcome this, the node-disjoint path with minimum hop count is considered in the proposed method. Minimum hop counts reduce the number of link sharing with neighbors and avoid the early depletion of the battery energy. A single optimal path model from a multi-objective function has been derived to find a solution based on the idea given in [34]. It is a challenging task to evaluate a multi-objective optimization. Therefore, a single objective function $F(x)$ has been set up based on energy consumption, hop counts, and traffic load. The multi-objective function based on weighted sum approach is formulated as,

$$
\begin{array}{r}
F(x)=\min \left(\alpha_{1} \alpha_{2} \frac{E(x)}{\beta}+\left(1-\alpha_{1}\right) \alpha_{2} H(x)+\left(1-\alpha_{2}\right) T(x)\right) \\
0 \leq \alpha_{1}, \alpha_{2} \leq 1
\end{array}
$$

The parameters $\alpha_{1}$ and $\alpha_{2}$ are weights and to normalize the three different parameters, a normalization coefficient $\beta$ is introduced and computed by taking average of $\beta_{1}$ and $\beta_{2}$, i.e., $\beta=\frac{\beta_{1}+\beta_{2}}{2}$.

The optimum value of $F(x)$ depends on the selection of weight value. $E(x)$ is total energy consumption (mJ) in the path between source and destination. $H(x)$ is the total hop counts in the path. $T(x)$ is total traffic load (packets/second) i.e., sum of the traffic queue of the nodes in the path. $x$ denotes the node-disjoint path. The term $\beta_{1}$ is the product of maximum used energy consumption in one hop and $H(x)$. The term $\beta_{2}$ is the product of maximum used energy consumption for one traffic load and $T(x)$.

Considering energy consumption in a path $E(x)$ is $3.5 \mathrm{~J}$, and number of hops $H(x)=3$, then $\frac{E(x)}{H(x)}=\frac{3.5 \mathrm{~J}}{3}=1.16 \mathrm{~J}$, and maximum energy value in one hop is taken as $1.2 \mathrm{~J}$. Therefore, $\beta_{1}$ is $1.2 \mathrm{~J}$ multiplied by the total number of hops. i.e., $\beta_{1}=3.6 \mathrm{~J} . \beta_{2}$ is also evaluated as that of $\beta_{1}$ by multiplying the total traffic load with the energy consumed for one traffic. The final node-disjoint multipaths are chosen after validating with Equation (1). We always try to keep three paths for data transfer. Hence, the resultant multipath selection for enhancing the performance is chosen by balancing the three objectives based on Equation (1) for the values of $\alpha_{i}$ greater than zero and less than 1 . The reason for the selection is explained in the following section.

\section{Exceptions}

1. In Equation (1), if the value of $\alpha_{1}$ is zero and $\alpha_{2}$ is one, $F(x)$ is a function of only hop counts. Energy consumption and traffic load of the participating nodes may be high in the selected path. Therefore, this condition is ignored.

2. If the value of $\alpha_{2}$ is zero, irrespective of $\alpha_{1}, F(x)$ is purely a function of only traffic load. This condition also becomes invalid. 
3. If the values of $\alpha_{1}$ and $\alpha_{2}$ are one, $F(x)$ is solely dependent on Energy consumption. This condition also becomes invalid. By keeping the exceptions in mind, weight parameter is considered to be $\alpha_{1}+\alpha_{2}=1$ and based on this, the paths are selected for data transfer.

\section{Proposed Multi-Objective Function-Based Node-Disjoint Multipath Routing Protocol}

In this section, we propose a multi-objective function-based routing protocol for performance enhancement. Multi-objective consideration is to prolong the lifetime of the network through energy saving. To avoid delay in finding the node-disjoint path at the destination, a time limit is defined to receive the RREQ. The RREQ packets reaching the destination after the set time limit are discarded without processing. To preserve network connectivity, a path maintenance procedure is introduced that begins to discover the paths once the route cache is filled with a single backup path to remove the network partitioning. Section 3.1 presents the assumptions and symbols used in the design and Section 3.2 deals with energy consumption computation and also presents a node-disjoint path formation procedure that discovers various paths based on the multi-objective function by eliminating the routes that consume high energy, which have a higher number of hops, and traffic load. The last Section 3.3 explains the simple path maintenance procedure.

\subsection{Assumptions and Notation}

The symbols used in the design are defined in Table 1.

Table 1. Notations used.

\begin{tabular}{ll}
\hline Symbol & Meaning \\
\hline$E C_{t x}$ & Energy consumed in transmitting the data \\
$E C_{r x}$ & Energy consumed in receiving data \\
$E C_{\text {sleep }}$ & Energy consumed in the sleep state \\
$E C_{\text {trans }}$ & Energy consumed for transition \\
$E C_{p r}$ & Energy consumed in processing \\
$E C_{q u}$ & Energy consumed in queuing of packets \\
$\mathrm{N}$ & Number of nodes in the path \\
$P_{n}$ & Number of packets \\
\hline
\end{tabular}

1. The transmit power level is chosen as $200 \mathrm{~mW}(23 \mathrm{dBm})$.

2. Nodes are anticipated to synchronize in a distributed way in energy save mode [35].

3. The transmission range of all the nodes is fixed.

4. The number of neighbors is kept as 10 [36], as the transfer of data and control packets is more costly than the link reordering, which is not done here. This paper is mainly focused on creating the node-disjoint path between the source and destination, so that we assumed that each node has at least 10 neighborhoods to ensure link availability and effective formation of the routing.

5. It is assumed that there is no interference between the nodes in different paths to enhance efficiency. In this proposed method, the node-disjoint path selection is introduced, in which the selected relay node has less link sharing with another path. As a result, it reduces the interference and enhancing the efficiency in terms of energy consumption and packet delivery ratio.

6. Each path is maintained to have a hop count between 5 and 10 [37] to boost the throughput.

\subsection{Energy Consumption Computation}

An analysis of Energy Consumption (EC) is given in this section. We first compute the energy consumption in a link between two nodes $i$ and $j\left(E C_{i, j}\right)$ as specified in Equation (2). $E C_{i}$ and $E C_{j}$ are found out as in Equations (3) and (4). Equation (5) gives the energy 
consumption for transmitting the entire data $\left(E C_{T}\right)$ in the link $i, j$. It is assumed that the total size of data is known priory. For all the $N-1$ links denoted as $(E(x))$, the total energy consumption of a path between source and destination is computed as given in Equation (6). $N$ represents the number of nodes in the path.

$$
\begin{gathered}
E C_{i, j}=E C_{i}+E C_{j} \\
E C_{i}=E C_{t x}+E C_{p r}+E C_{q u} \\
E C_{i, j}=(1-\Delta)\left(E C_{r x}+E C_{\text {sleep }}\right)+E C_{r x}+E C_{\text {sleep }}+E C_{\text {trans }}
\end{gathered}
$$

where $\Delta$ represents the number of neighbors in each hop, $E C_{r x}, E C_{\text {sleep }}$ and $E C_{\text {trans }}$ are energy consumption in receiving the data packet, sleep and transition from sleep, respectively. $(1-\Delta) \times\left(E C_{r x}+E C_{\text {sleep }}\right)$ represents the energy due to overhearing nodes.

$$
E C_{T}=\sum_{s=1}^{P_{n}} E C_{i, j}(s)
$$

where $P_{n}=\frac{\text { Data size }}{\text { Packet size }}$

$$
E(x)=\sum_{n=1}^{N-1} E C_{T}(n)
$$

We can thus select the path that has minimum energy consumption for data transfer using Equations (2)-(6).

\section{Formation of Node-Disjoint Path}

After finding node-disjoint paths that fulfill the objective function given in Equation (1) for reliable transfer, multiple paths for data transfer are found. The destination after the defined time limit responds with a RREP to the source node after validating the collected paths for node-disjoint. Destination provides all node-disjoint routes to the source node as route responses. The source node only obtains the RREP from the intended destination based on the ID. Source discards the RREPs received from the intermediate nodes. The node-disjoint paths (ND) are represented as a square matrix of size $(I \times J)$ as given in [25], in which each element in the path is found as specified in Equation (7).

$$
N D_{I \times J}=\left\{\begin{array}{cc}
1 & \text { If } \text { Ith and Jth paths have } \\
0 & \text { at least a common node }
\end{array}\right.
$$

In Figure 1, node 1 is the source and node 7 is the destination. There are three possible paths exists between the node 1 and node 7 . Number of paths determines the size of the node-disjoint matrix; therefore, the matrix size is going to be $3 \times 3$. The element of node-disjoint matrix $3 \times 3$ is formed by comparing the existing paths. The first path is compared with the remaining two paths to form the first-row elements, second path with the third and first path for the second-row elements and third with first and second path, respectively, for the third-row elements. For the above three paths, the matrix is formed as shown below in Equations (8) and (9).

$$
\begin{gathered}
N D_{I \times J}=\left[\begin{array}{lll}
e_{11} & e_{12} & e_{13} \\
e_{21} & e_{22} & e_{23} \\
e_{31} & e_{32} & e_{33}
\end{array}\right] \\
N D_{I \times J}=\left[\begin{array}{lll}
0 & 1 & 0 \\
1 & 0 & 0 \\
0 & 0 & 0
\end{array}\right]
\end{gathered}
$$


From Figure 1, Path 1 and Path 2 have the common node (node 5), so node-disjoint matrix element $e_{12}$ and $e_{21}$ are one. In addition, the remaining elements $\left(e_{11}, e_{13}, e_{22}, e_{23}, e_{31}, e_{32}, e_{33}\right)$ set to zero. This is because of there is no common node exists between the Path 2 and Path 3 , similarly Path 1 and Path 3 . All the diagonal elements are zero, due to the comparison of the first path with first path, second by second and so on. Based on Equation (8), the node-disjoint path is the third row containing all zeros in the matrix. Thus, the Path 3 (i.e., node $1 \rightarrow$ node $4 \rightarrow$ node $6 \rightarrow$ node 7 ) is selected to transfer the data between the source and destination. The aim is to eliminate the sharing of nodes in the chosen path to reduce the number of path breakages due to node sharing and to enhance the node lifetime.

The destination predicts the node-disjoint paths and gives back the route reply to the source node. The source node then selects multiple paths based on the objective function given in Equation (1). The collected multiple paths are sequenced based on the objective function value. At a time, three paths are chosen from this for data transfer. If the number of collected paths is less than three, or node-disjoint paths are not found, then a new route discovery is started. The main advantage of using node-disjoint path selection is that during data transmission, the node will not fail due to overloaded traffic.

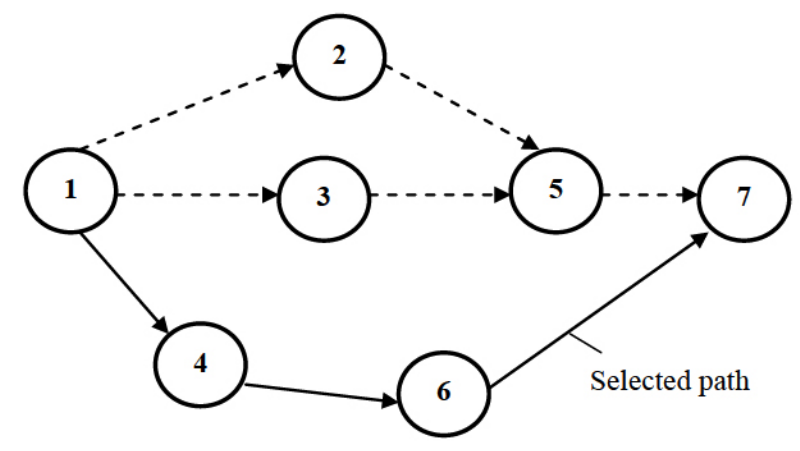

(a)

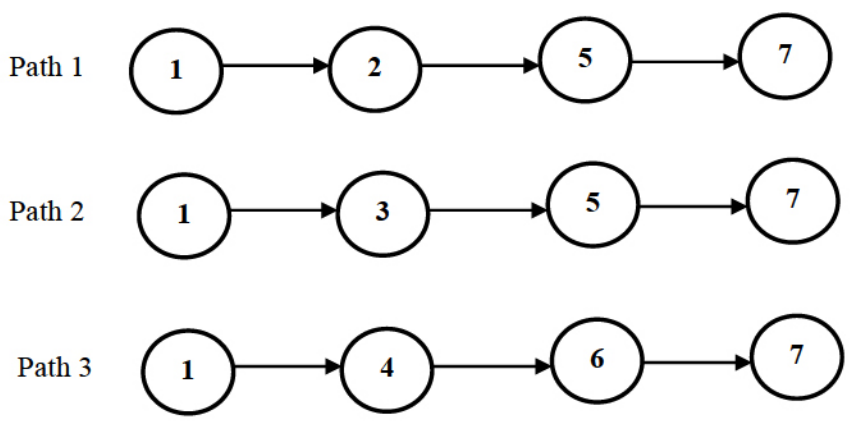

(b)

Figure 1. Example for path selection: (a) node arrangement (b) path possibilities between the source and destination.

\subsection{Path Maintenance}

The source after validation maintains three to ten multiple paths. Three paths are selected for data transfer. Path maintenance will be activated once a node detects that the next hop node to the destination is failed in the data transmission phase. The path maintenance is done as follows,

Step 1: Once the forwarding node detects a failure in the next hop link, it informs the source by means of a unicast message i.e., Route Error (RERR). 
Step 2: The source after receiving the RERR, stops transmitting in the failed path. The data transfer will be done through a backup path chosen from the route cache. Once the route cache is empty, source initiates the route discovery process.

Step 3: After receiving the new RREP from the destination, the source validates for the objective function and then it transmits the data packets.

\section{Simulation Results and Discussion}

\subsection{Experimental Setup}

Performance of the proposed algorithm, MFNMR, existing DSR, HNNDPS, and MDSR are tested in several simulated scenarios. Diverse simulation scenarios have been created by varying number of nodes, node mobility, traffic load, and amount of pause time. Implementation of the proposed is done in C++-based NS-2 simulator [12].

The simulation parameters are listed in Table 2. In all scenarios, nodes are placed randomly in a $1000 \mathrm{~m} \times 1000 \mathrm{~m}$ region. The maximum transmit range of each mobile node is $250 \mathrm{~m}$. In general, nodes move according to the random way point mobility model [38] with a speed in the range $[0,10] \mathrm{m} / \mathrm{s}$. In this mobility model, all nodes move toward a new destination position and stay there for a specified time called pause time and again proceed towards a new direction.

Table 2. Simulation setup.

\begin{tabular}{cc}
\hline Parameter & Value \\
\hline Network Area & $1000 \mathrm{~m} \times 1000 \mathrm{~m}$ \\
Transmission Range (R) & $250 \mathrm{~m}$ \\
Data Size & $5 \mathrm{Mbytes}$ \\
Data Rate & $2 \mathrm{Mbits} / \mathrm{s}$ \\
Initial Energy & $180 \mathrm{~J}$ \\
Maximum Node Speed & $10 \mathrm{~m} / \mathrm{s}$ \\
Number of Nodes & $100-150$ \\
Packet Size & 256 bytes \\
Pause Time & $0-600 \mathrm{~s}$ \\
Simulation Time & $900 \mathrm{~s}$ \\
Traffic Load & $1-5$ packets \\
Traffic Type & CBR \\
\hline
\end{tabular}

The channel capacity is $2 \mathrm{Mbps}$ and MAC protocol uses IEEE 802.11 power save mode. The ad hoc traffic indication mode window size and beacon interval is set at $0.05 \mathrm{~s}$ and $0.25 \mathrm{~s}$. Each simulation is run for $900 \mathrm{~s}$ each time. The propagation model used is two ray ground model. The analysis involves an average number of control messages, energy consumption, packet delivery ratio, network lifetime, and latency. Simulation results were taken after 10 runs to obtain steady state value.

\subsection{Result Analysis}

\subsubsection{Impact on Number of Nodes}

Figures 2-6 show the performance analysis of the MFNMR design for different network sizes by keeping the pause time as $300 \mathrm{~s}$ and packet rate as 2 packets/s.

As shown in Figure 2 the proposed method consumes significantly less energy than all the other methods. The reason behind this reduction is that the data transfer is via the selected path that consumes less energy, less hops, and traffic. In addition, also energy is proportional to the hops. Therefore, optimizing the hop counts, traffic load leads to less energy consumption. 


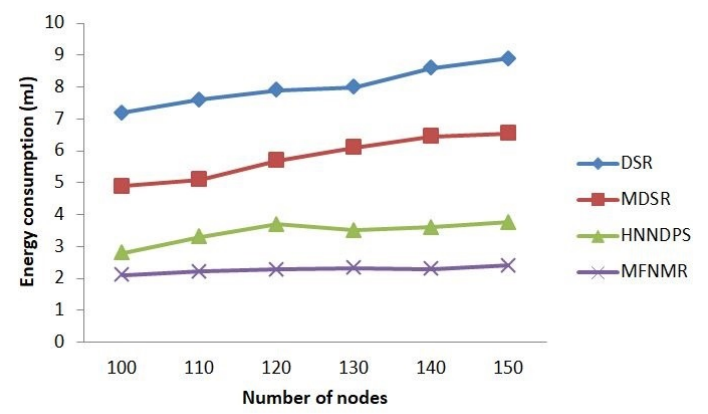

Figure 2. Energy Consumption Analysis based on Number of Nodes.

Packet delivery ratio of the proposed protocol with respect to the network size is given in Figure 3. It is observed that the proposed MFNMR has better PDR than DSR, MDSR and HNNDPS. The PDR increases when the number of nodes increases. The increased number of nodes ensures that the optimum path between the source and destination is chosen. It also increases the number of backup paths and the longevity i.e., path lifetime.



Figure 3. PDR Analysis based on Number of Nodes.

Average packet latency for the proposed system is shown in Figure 4 for various network sizes. It is seen that the proposed MFNMR achieves a less delay in comparison with all the other methods. The increasing number of paths between the nodes leads to select the best path which has less hop counts and no sharing of nodes. So that selected path has a lower latency and makes better use of bandwidth.

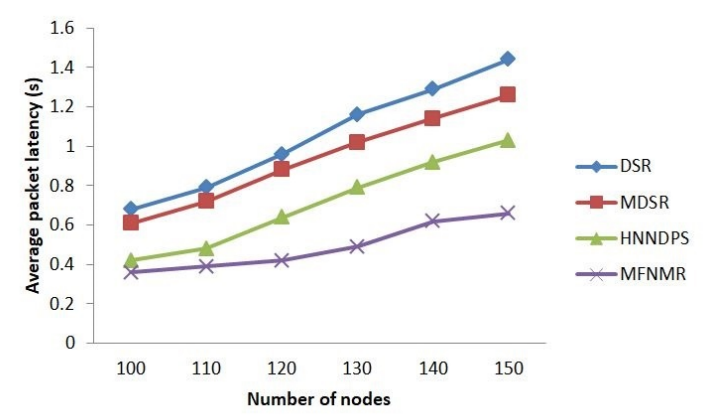

Figure 4. Average Packet Latency Analysis based on Number of Nodes.

Figure 5 shows the control messages for the MFNMR design for various network sizes. It indicates that the overhead increases as the number of nodes increases. This is because of the flooding technique used in the network to find the paths between the source and destination. 




Figure 5. Routing Overhead Analysis based on Number of Nodes.

Path lifetime in s is shown in Figure 6. Path lifetime is measured as the time duration between the starting of data transfer in a particular path and any one of the nodes in the path is found to be dead. Path lifetime in the proposed MFNMR is better compared to all. The reason behind this is that the path selection is based on multi-objective function. The node with high energy consumption and traffic queue will not take part in data transfer. High traffic load will result in packet loss and it leads to unnecessary energy consumption. HNNDPS also shows an improved path lifetime compared to MDSR and DSR because of the consideration of reliable paths. MDSR is better than the basic DSR.

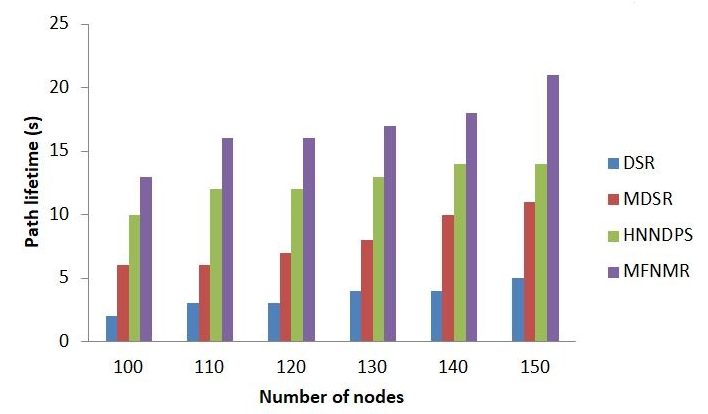

Figure 6. Path Lifetime Analysis based on Number of Nodes.

\subsubsection{Impact on Node's Speed}

Figures 7-10 show the performance analysis of the MFNMR design for various node speeds by keeping the pause time as $300 \mathrm{~s}$ and packet rate as 2 packets/s.

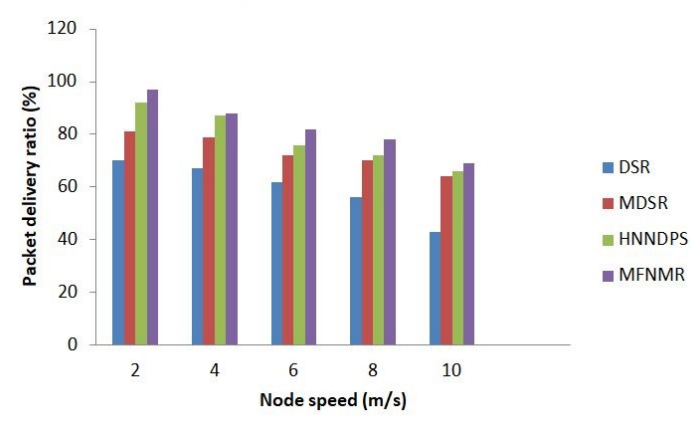

Figure 7. PDR Analysis based on Node Speed.

Figure 7 shows the effect of mobility on packet delivery ratio. In the case of MDSR and DSR, there are possibilities that some of the links might be shared by more than one shortest path causing increased traffic on those links. This may lead to congestion and hence data packets transmitted through these links may face additional delay and PDR will reduce. In the proposed MFNMR, due to the consideration of minimum energy, hop counts, and traffic in path selection, the path breakage is minimized leading to a higher 
PDR compared to all the other methods. When the mobility increases, paths between source and destination may break often leading to the selection of alternate path. The PDR value for the proposed is varied nearly from $98 \%$ to $70 \%$ when the mobility of node increases. From these, it can be said that the system is stable even in mobility condition.

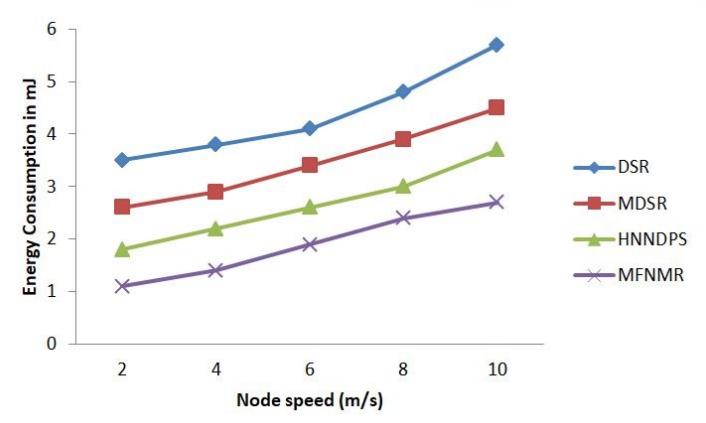

Figure 8. Energy Consumption Analysis based on Node Speed.

It is observed from the Figure 8 that the proposed MFNMR consumes less energy than DSR, MDSR and HNNDPS. The proposed MFNMR has high link stability and has less hop count than all the other methods. MFNMR reduces the packet loss and retransmission resulting in reduced energy consumption than DSR, MDSR and HNNDPS.

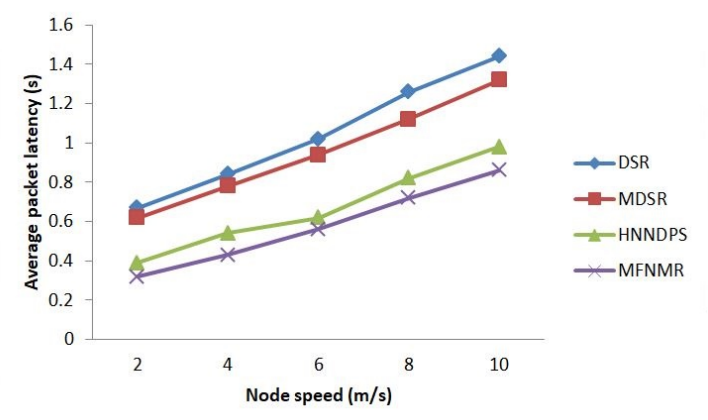

Figure 9. Average Packet Latency Analysis based on Node Speed.

It is noted from the Figure 9 that the proposed MFNMR has less average packet latency. This is because of the proposed MFNMR has more backup paths than DSR, MDSR and HNNDPS. Additionally, the proposed method considers the traffic loads and hop count to select the best path, therefore it ensures the shortest path to reach the destination and reduces the delay in the packet delivery.

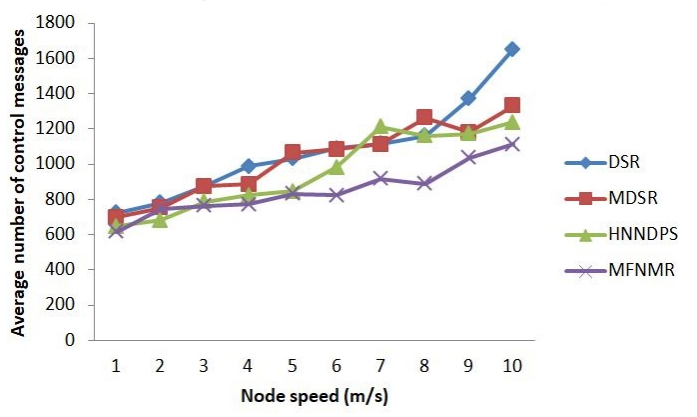

Figure 10. Routing Overhead Analysis based on Node Speed.

The routing overhead is an important metric for measuring the efficiency in terms of node battery power consumption and for increasing the probability of packet collision and delay. Figure 10 shows the routing overhead for different node speeds for all the 
methods. Among all, proposed method generates less overhead compared to DSR, MDSR, and HNNDPS. It is seen that the control messages are increasing from 634 to 1189 for MFNMR for the node speed of 1 to $10 \mathrm{~m} / \mathrm{s}$, whereas for other methods it is higher. This is due to the involvement of fewer nodes in the selected paths. In addition, also the node failure is less because of the consideration of the traffic queue in node selection.

\subsubsection{Impact on Network Traffic}

Figures 11-14 show the performance analysis of the MFNMR design for various traffic load by keeping the pause time as $300 \mathrm{~s}$ and number of nodes as 100 .

Figure 11 represents the packet delivery ratio analysis for various traffic loads from 1 to 5 packets/s for a network of 100 nodes. In the case of MDSR and DSR, there are possibilities that some of the links might be shared by more than one shortest path causing increased traffic on those links. This may lead to congestion and hence data packets transmitted through these links may face additional delay and PDR will reduce. In MFNMR, due to the consideration of minimum energy, hop, and traffic in path selection, the path breakage is minimized leading to a higher PDR compared to all the other methods.

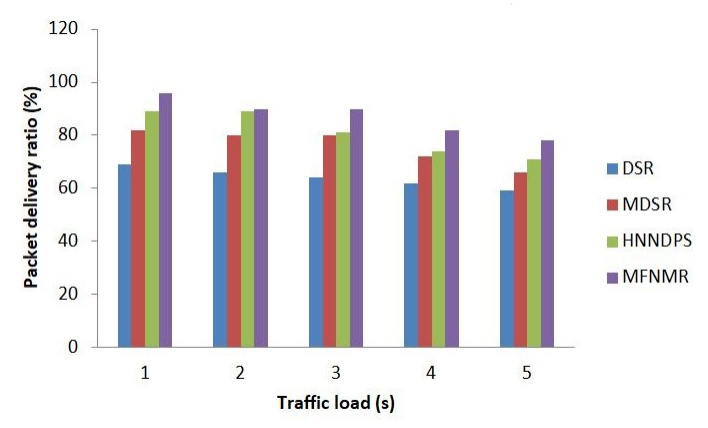

Figure 11. PDR Analysis based on Network Traffic.

Figure 12 represents the average latency of MFNMR in comparison with DSR, MDSR, and HNNDPS designs. It is seen that the design MFNMR achieves a lesser delay in comparison with all the other methods. However, in DSR and other methods, if many traffic connections start sharing few links, it leads to scarcity of available bandwidth and intermediate link failure. Since, the MFNMR design has hop as one of the objectives and there is no sharing of nodes in the selected paths, it leads to less latency with effective use of bandwidth. In addition, path maintenance makes the system to work efficiently with less delay.

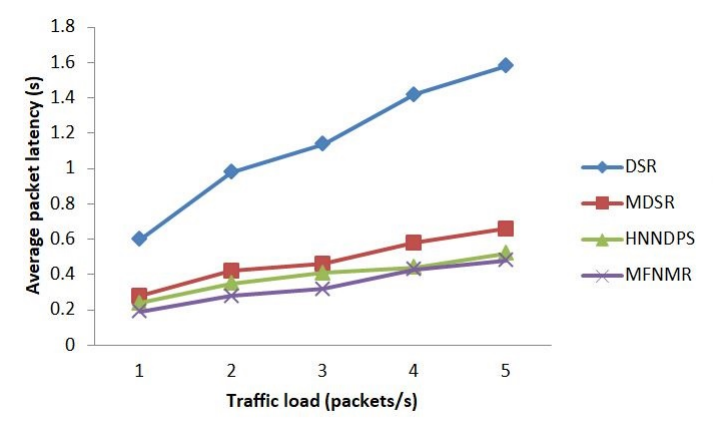

Figure 12. Average Packet Latency Analysis based on Network Traffic.

Energy consumption in $\mathrm{mJ}$ of the proposed MFNMR is shown in Figure 13. The proposed MFNMR is better compared to all in terms of energy consumption. The reason behind this is that the path selection is based on multi-objective function. The energy consumption increases as the traffic load increases. High traffic load will result in packet loss and it leads to increase energy consumption. 


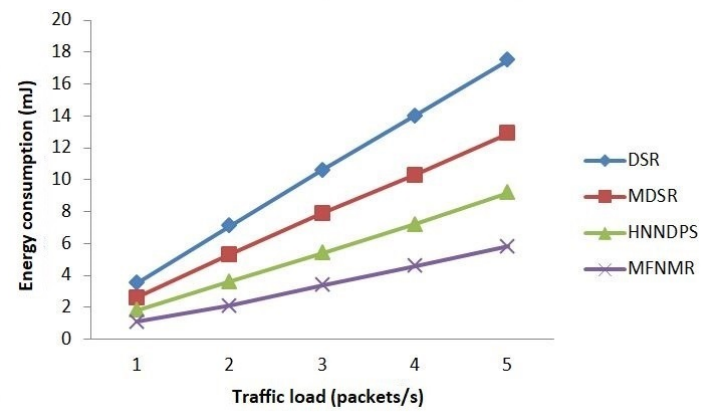

Figure 13. Energy Consumption Analysis based on Network Traffic.

Figure 14 shows the average control overhead for different traffic loads. The proposed method generates less overhead compared to DSR, MDSR, and HNNDPS. This is because of the involvement of fewer nodes in the selected paths with no sharing nodes. In addition, also the node failure is less due to the consideration of the traffic queue in node selection. However, high traffic load will result in packet loss and it leads to packet retransmission and control overhead.



Figure 14. Routing Overhead Analysis based on Network Traffic.

\subsubsection{Impact on Pause Time}

Figures 15-18 show the performance analysis of the MFNMR design with pause time by keeping the traffic load 2 packets/s and number of nodes as 100 .

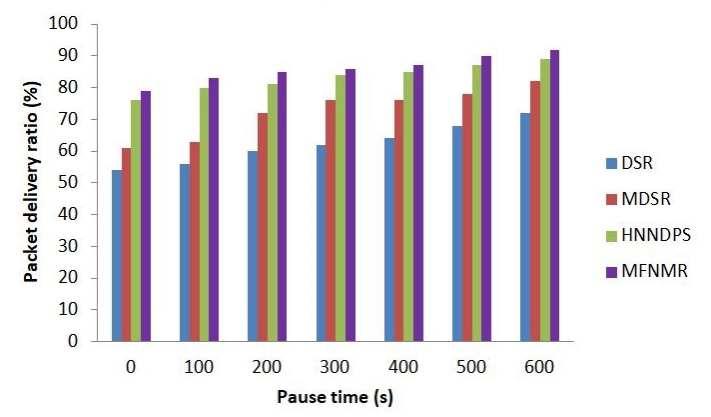

Figure 15. PDR Analysis based on Pause Time.

The analysis of PDR with respect to pause time is shown in Figure 15. The proposed design MFNMR achieves better PDR value than DSR, MDSR, and HNNDPS. It is noted that the proposed design MFNMR achieves a PDR value of nearly $80 \%$ even at the pause time of $0 \mathrm{~s}$. In MFNMR, the backup paths can be selected from the route cache effectively. Additionally, the increased pause time ensures the link stability and path lifetime. Hence, data can be transmitted as they are received without making them wait in the queues. As a result, MFNMR reduces the packet loss and increases the PDR. 


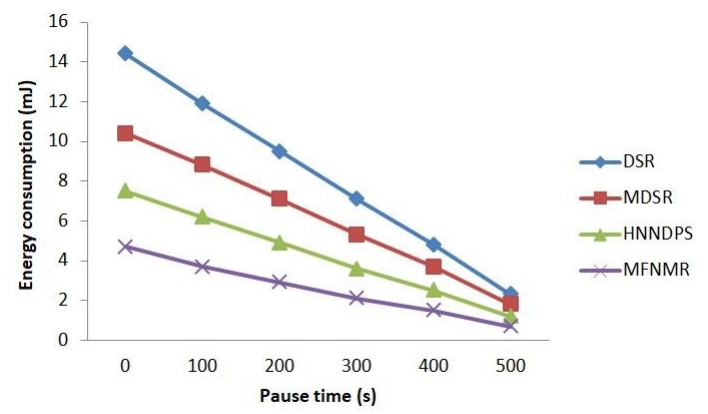

Figure 16. Energy Consumption Analysis based on Pause Time.

The analysis of the energy consumption in $\mathrm{mJ}$ of the proposed MFNMR with respect to the pause time is shown in Figure 16. The proposed MFNMR shows less energy consumption than DSR, MDSR, and HNNDPS. Figure 16 shows that when the stop duration of the nodes increases, the total energy spent by the nodes decreases, implying that pause time is inversely related to total energy consumed by the nodes. The average rate of energy consumption by each node decreases as total energy consumption lowers.

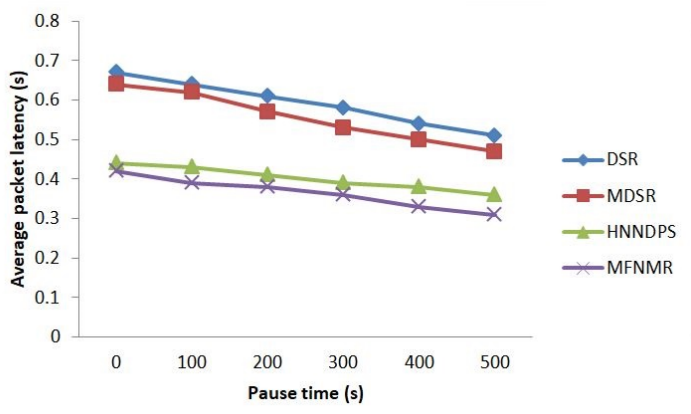

Figure 17. Average Packet Latency Analysis based on Pause Time.

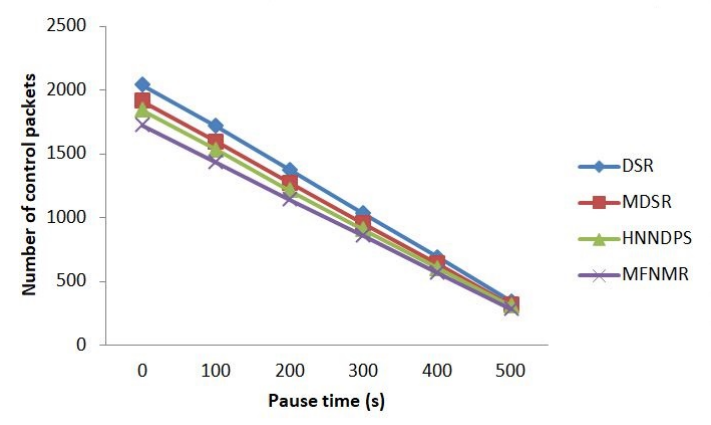

Figure 18. Routing Overhead Analysis based on Pause Time.

It is observed from Figures 17 and 18 that the average packet latency and control overhead decrease when the pause time increases. From the simulation results, the MFNMR provides better link stability and avoids the packet loss. Additionally, it increases the path lifetime and number of backup paths. As a result, MFNMR has a less latency in packet delivery and control overhead than DSR, MDSR and HNNDPS.

The available backup paths at the source node are analyzed for all the methods for a network of 200 nodes and it is shown in Figure 19. It is seen that as the time is increasing, due to node mobility, the available paths are reduced in all the methods. After $80 \mathrm{~s}$, the performance of MDSR and HNNDPS are same. Initially, the proposed has 9 paths as backup, after $40 \mathrm{~s}$, the number is gradually decreasing. This shows that using the proposed method, the backup paths can be selected from the route cache effectively. Hence, data can be transmitted as they are received without making them wait in the queues. 


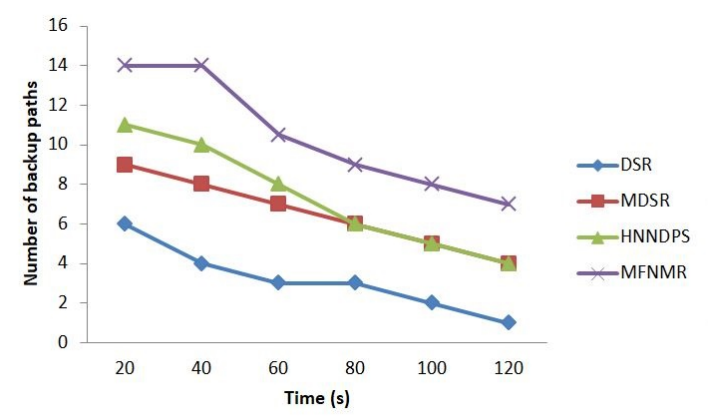

Figure 19. Backup Paths Analysis based on Time.

The performance analysis is shown in Table 3 for a network of 300 nodes. The analysis consists of the following parameters: traffic load is 2 packets $/ \mathrm{s}$, node speed is $4 \mathrm{~m} / \mathrm{s}$ and pause time is $300 \mathrm{~s}$.

Table 3. Performance analysis of DSR, MDSR, HNNDPS and MFNMR.

\begin{tabular}{lllllll}
\hline Methods & PDR (\%) & Latency (s) & $\begin{array}{l}\text { Energy } \\
\text { Consumption }(\mathbf{m J})\end{array}$ & $\begin{array}{l}\text { No. of Available } \\
\text { Backup Paths }\end{array}$ & $\begin{array}{l}\text { Number of } \\
\text { Control Packets }\end{array}$ & Path Lifetime (s) \\
\hline DSR [12] & 68 & 1 & 7 & 6 & 1020 & 2 \\
MDSR [19] & 80 & 0.5 & 5 & 7 & 951 & 6 \\
HNNDPS [18] & 89 & 0.42 & 3 & 9 & 906 & 10 \\
MFNMR & 90 & 0.35 & 2.2 & 9 & 856 & 12 \\
\hline
\end{tabular}

As far as PDR is concerned, proposed approach MFNMR achieves 1.13\% higher than HNNDPS, $12.5 \%$ higher than MDSR, 32\% higher than DSR. Latency for the proposed is $35 \%$, $30 \%$, and $16.5 \%$ less than DSR, MDSR, and HNNDPS, respectively. The energy consumption for the proposed is $68.5 \%, 56 \%$, and $26.7 \%$ less than DSR, MDSR, and HNNDPS. It is seen that the number of available backup paths for the proposed system is more than 2.3 times than DSR, 1.55 times than MDSR, 1.27 times than HNNDPS.

It is seen that the routing overhead for the proposed is $16.1 \%, 10 \%$, and $5.5 \%$ less than that of DSR, MDSR, and HNNDPS. The reason behind is that it involves optimal path selection and maintenance. Node energy depletion due to insufficient energy is almost eliminated in the proposed because of the multi-objective optimization in path selection approach. Path lifetime also attains a higher compared to all the other methods. This is because of the presence of node-disjoint and multi-objective function-based path selection in the proposed method.

The performance of the multipath design MFNMR is also analyzed by comparing it with the previously proposed techniques PDSEER [25], EE-BPS [28], and OPSM [29] and it is given in Table 4 for a network of 100 nodes. For this analysis, traffic load is maintained as 2 packets/s and pause time as $300 \mathrm{~s}$.

Table 4 shows that the overall energy consumption in MFNMR is reduced to a significant amount compared to other proposed schemes. Control messages are $32.25 \%, 16 \%$, and 16\% lower when compared to PDSEER, EE-BPS and OPSM, respectively. A higher packet delivery ratio is achieved than all the other methods. The reason is that the use of multi-objective function-based path selection avoids path breakages. The lifetime of the route is retained as that of the OPSM, but greater than the transmit power controlbased PDSEER and EE-BPS methods. The control messages for finding multiple paths in the MFNMR design are more than that of other proposed techniques. This is due to the proposed MFNMR approach uses more control packets for finding multiple paths. 
Table 4. Performance analysis of PDSEER, EE-BPS, OPSM and MFNMR.

\begin{tabular}{lllllll}
\hline Methods & $\begin{array}{l}\text { Number of } \\
\text { Control Messages }\end{array}$ & $\begin{array}{l}\text { Energy } \\
\text { Consumption (mJ) }\end{array}$ & PDR (\%) & Latency (s) & Path Lifetime (s) & $\begin{array}{l}\text { Number of } \\
\text { Path Breakage }\end{array}$ \\
\hline PDSEER [25] & 220 & 3.1 & 90 & 0.48 & 10 & 24 \\
EE-BPS [28] & 216 & 2.5 & 91 & 0.42 & 11 & 24 \\
OPSM [29] & 221 & 2.5 & 92 & 0.38 & 12 & 20 \\
MFNMR & 278 & 2.1 & 93 & 0.32 & 12 & 18 \\
\hline
\end{tabular}

\section{Conclusions}

MFNMR is proposed mainly to transfer the data efficiently between source and destination and to prolong the network lifetime. It is observed that the proposed approach MFNMR achieves 1.13\% higher than HNNDPS, 12.5\% higher than MDSR, 32\% higher than DSR. Latency for the proposed is 35\%, 30\%, and 16.5\% less than DSR, MDSR, and HNNDPS, respectively. The energy consumption for the proposed is $68.5 \%, 56 \%$, and $26.7 \%$ less than DSR, MDSR, and HNNDPS. Additionally, the routing overhead for the proposed is $16.1 \%, 10 \%$, and $5.5 \%$ less than that of DSR, MDSR, and HNNDPS. This algorithm can be applied to communication problems for material - embedded sensing devices in loose and unreliable coupling networks of low-resource computing nodes with restricted energy. MFNMR can be appropriate for power harvesting in structural health monitoring applications where they can provide energy to remote computing systems for processing the data and provide battery operated systems with longevity.

Author Contributions: Conceptualization, B.V. and K.K.; methodology, B.V. and K.K.; software, B.V. and K.K.; validation, B.V., K.K., D.S., R.N.A. and J.C.; formal analysis, B.V., K.K., D.S., R.N.A.; writing-original draft preparation, B.V. and K.K.; writing—review and editing, D.S., R.N.A. and J.C.; funding acquisition, J.C. All authors have read and agreed to the published version of the manuscript.

Funding: This research received no external funding.

Acknowledgments: This work was supported Korea Environmental Industry \& Technology Institute (KEITI) grant funded by the Korea government (Ministry of Environment). Project No. RE202101551, the development of IoT-based technology for collecting and managing Big data on environmental hazards and health effects.

Conflicts of Interest: The authors declare no conflict of interest.

\section{References}

1. Perkins, C.E. Ad Hoc Networking; Addison-Wesley: Boston, MA, USA, 2001.

2. Jahir, Y.; Atiquzzaman, M.; Refai, H.; Anirudh Paranjothi, A.; Presti, P.G.L. Routing protocols and architecture for disaster area network: A survey. Ad Hoc Netw. 2019, 82, 1-14. [CrossRef]

3. Kanellopoulos, D.; Sharma, V.K. Survey on Power-Aware Optimization Solutions for MANETs. Electronics 2020,9 , 1129. [CrossRef]

4. Muchtar, F.; Abdullah, A.H.; Al-Adhaileh, M.; Zamli, K.Z. Energy conservation strategies in Named Data Networking based MANET using congestion control: A review. J. Netw. Comput. Appl. 2020, 152, 102511. [CrossRef]

5. Farkhana, M.; Hanan, A.A.; Suhaidi, H.; Tajudin, K.A.; Zuhairi, Z.K. Energy conservation of content routing through wireless broadcast control in NDN based MANET: A review. J. Netw. Comput. Appl. 2019, 131, 109-132. [CrossRef]

6. Abid, M.A.; Belghith, A.; Drira, K. SARP: A dynamically readjustable period size proactive routing protocol for MANETs. J. Comput. Syst. Sci. 2015, 81, 496-515. [CrossRef]

7. Kunz, T.; Alhalimi, R. Energy-efficient proactive routing in MANET: Energy metrics accuracy. Ad Hoc Netw. 2010, 8, 755-766. [CrossRef]

8. Chatterjee, S.; Das, S. Ant colony optimization based enhanced dynamic source routing algorithm for mobile Ad-hoc network. Inf. Sci. 2015, 295, 67-90. [CrossRef]

9. Darabkh, K.A.; Alfawares, M.G.; Althunibat, S. MDRMA: Multi-data rate mobility-aware AODV-based protocol for flying ad-hoc networks. Veh. Commun. 2019, 18, 100163. [CrossRef]

10. Tarique, M.; Tepe, K.E.; Adibi, S.; Erfani, S. Survey of multipath routing protocols for mobile ad-hoc networks. J. Netw. Comput. Appl. 2009, 32, 1125-1143. [CrossRef] 
11. Gomes, T.; Martins, L.; Ferreira, S.; Pascoal, M.; Tipper, D. Algorithms for determining a node-disjoint path pair visiting specified nodes. Opt. Switch. Netw. 2017, 23, 189-204. [CrossRef]

12. Ali, H.A.; Areed, M.F.; Elewely, D.I. An on-demand power and load-aware multi-path node-disjoint source routing scheme implementation using NS-2 for mobile ad-hoc networks. Simul. Model. Pract. Theory 2018, 80, 50-65. [CrossRef]

13. Hou, A.; Wu, C.Q.; Fang, D.; Wang, Y.; Wang, M. Bandwidth scheduling for big data transfer using multiple fixed node-disjoint paths. J. Netw. Comput. Appl. 2017, 85, 47-55. [CrossRef]

14. Bhattacharya, A.; Sinha, K. An efficient protocol for load-balanced multipath routing in mobile ad hoc networks. Ad Hoc Netw. 2017, 63, 104-114. [CrossRef]

15. Tsirigos, A.; Haas, Z.J.; Tabrizi, S.S. Multipath routing in mobile ad hoc networks or how to route in the presence of frequent topology changes. In Proceedings of the Military Communications Conference, Vienna, VA, USA, 28-31 October 2001; Volume 2, pp. 878-883.

16. Ren, J.; Zhang, Y.; Zhang, K.; Liu, A. Lifetime and Energy Hole Evolution Analysis in Data-Gathering Wireless Sensor Networks. IEEE Trans. Ind. Inform. 2014, 12, 788-800. [CrossRef]

17. Papadimitratos, P.; Haas, Z.J.; Sirer, E.G. Path set selection in mobile ad hoc networks. In Proceedings of the Third ACM Symposium on Mobile ad hoc Networking and Computing, Lausanne, Switzerland, 9-11 June 2002; pp. 1-11.

18. Hemmati, E.; Sheikhan, M. Hopfield neural network for disjoint path set selection in mobile ad-hoc networks. In Proceeding of the 6th IEEE International Conference on Wireless Communication and Sensor Networks, Allahabad, India, 15-19 December 2010; pp. 1-5.

19. Nasipuri, A.; Das, S.R. On-demand multipath routing for mobile ad hoc networks. In Proceedings of the 8th International Conference on Computer Communications and Networks, Boston, MA, USA, 11-13 October 1999; pp. 64-70.

20. Wisitpongphan, N.; Tonguz, O.K. Disjoint multipath source routing in ad hoc networks: Transport capacity. In Proceedings of the IEEE 58th Vehicular Technology Conference (VTC), Orlando, FL, USA, 6-9 October 2003; Volume 4, pp. $2207-2211$.

21. Karkvandi, H.R.; Pecht, E.; Yadid, O. Effective lifetime-aware routing in wireless sensor networks. IEEE Sens. J. 2011, 11, 3359-3367. [CrossRef]

22. Yadav, A.K.; Das, S.K.; Tripathi, S. EFMMRP: Design of efficient fuzzy based multi-constraint multicast routing protocol for wireless ad-hoc network. Comput. Netw. 2017, 117, 15-23. [CrossRef]

23. Ravi, G.; Kashwan, K.R. A new routing protocol for energy efficient mobile applications for ad hoc networks. Comput. Electr. Eng. 2015, 48, 77-85. [CrossRef]

24. Taha, A.; Alsaqour, R.; Uddin, M.; Abdelhaq, M.; Saba, T. Energy Efficient Multipath Routing Protocol for Mobile Ad-Hoc Network Using the Fitness Function. IEEE Access 2017, 5, 10369-10381. [CrossRef]

25. Bhanumathi, V.; Dhanasekaran, R. Path discovery and selection for energy efficient routing with transmit power control in MANET. Malays. J. Comput. Sci. 2013, 26, 124-139.

26. Ahmed, I. Reliable coverage area based link expiration time (LET) routing metric for mobile ad hoc networks. Ad Hoc Netw. 2010, $28,466-476$.

27. Sahu, R.K.; Chaudhari, N.S. Energy Reduction Multipath Routing Protocol for MANET Using Recoil Technique. Electronics 2018, 7, 56. [CrossRef]

28. Bhanumathi, V.; Dhanasekaran, R. Energy Efficient Routing with Transmission Power Control based Biobjective Path Selection Model for Mobile Ad-hoc Network. WSEAS Trans. Comput. 2011, 11, 407-417.

29. Bhanumathi, V.; Dhanasekaran, R. Efficient data transfer in mobile ad hoc network using OPSM for disaster response applications. J. Appl. Res. Technol. 2015, 13, 392-401. [CrossRef]

30. Haenggi, M.; Puccinelli, D. Routing in ad hoc networks: A case for long hops. IEEE Commun. Mag. 2005, 43, 93-101. [CrossRef]

31. Dargie, W.; Poellabauer, C. Network Layer, Fundamental of Wireless Sensor Networks Theory and Practice; Wiley: New York, NY, USA, 2010; pp. 163-204.

32. Baynast, A.D.; Mahonen, P.; Petrova, M. ARQ-based cross-layer optimization for wireless multicarrier transmission on cognitive radio networks. J. Comput. Netw. Int. J. Comput. Telecommun. Netw. 2008, 52, 778-794. [CrossRef]

33. Ponguwala, M.; Rao, S. E2-SR: A novel energy-efficient secure routing scheme to protect MANET-IoT. IET Commun. 2019, 13, 3207-3216. [CrossRef]

34. Kim, I.Y.; de Weck, O.L. Adaptive weighted sum method for multiobjective optimization: A new method for Pareto front generation. J. Struct. Multidiscip. Optim. 2006, 31, 105-116. [CrossRef]

35. Huang, L.; Lai, T.H. On the Scalability of IEEE 802.11 Ad-Hoc Networks. In Proceedings of the ACM Mobi Hoc, Lausanne, Switzerland, 9-11 June 2002; pp. 173-182.

36. Fall, K.; Varadhan, K. ns Nodes and Documents, The VINT Project, UC Berkeley, LBL, USC/ISI, and Xerox PARC. Available online: http:/ / www.isi.edu/ salehi/ns.doc/+ (accessed on 19 July 2021).

37. Ng, P.C.; Liew, S.C. Throughput Analysis of IEEE 802.11 Multi-hop Ad hoc Networks. IEEE/ACM Trans. Netw. 2007, 15, 309-322. [CrossRef]

38. Bettstetter, C.; Hartenstein, H.; Costa, X.P. Stochastic Properties of the Random Waypoint Mobility Model. J. Wirel. Netw. 2004, 10, 555-567. [CrossRef] 